\title{
Isolating auroral FUV emission lines using compact, broadband instrumentation.
}

P. M. Molyneux ${ }^{\mathrm{a}, *}$, N. P. Bannister ${ }^{\mathrm{a}}$, E. J. Bunce ${ }^{\mathrm{a}}$, D. Grodent ${ }^{\mathrm{b}}$, J. T. Clarke $^{\mathrm{c}}$, K. Fleury-Frenette $^{\mathrm{d}}$, J. -C. Gérard ${ }^{\mathrm{b}}$, J. Lapington ${ }^{\mathrm{a}}$, J. D. Nichols ${ }^{\mathrm{a}}$, E. Renotte ${ }^{\mathrm{d}}$, L. Rossi ${ }^{\mathrm{d}}$

${ }^{a}$ Department of Physics and Astronomy, University of Leicester, University Road, Leicester, UK

${ }^{b}$ LPAP, Institut d'Astrophysique et de Géophysique, Université de Liège, Liège, Belgium

${ }^{c}$ Center for Space Physics, Boston University, 957 Commonwealth Ave., Boston, MA 02215, USA

${ }^{d}$ Centre Spatial de Liège, Université de Liège, Liège, Belgium

\begin{abstract}
Images of auroral emissions at far ultraviolet (FUV, $122-200 \mathrm{~nm}$ ) wavelengths are useful tools with which to study magnetospheric-ionospheric coupling, as the scattered sunlight background in this region is low, allowing both dayside and nightside auroras to be imaged simultaneously. The ratio of intensities between certain FUV emission lines or regions can be used to characterise the precipitating particles responsible for auroral emissions, and hence is a useful diagnostic of magnetospheric dynamics. Here, we describe how the addition of simple transmission filters to a compact broadband imager design allows far ultraviolet emission ratios to be deduced while also providing large-scale instantaneous images of the aurora. The low mass and volume of such an instrument would make it well-suited for both small satellite Earth-orbiting missions and larger outer planet missions from which it could be used to characterise the tenuous atmospheres observed at several moons, as well as studying the auroral emissions of the gas giants. We present a study to investigate the accuracy of a technique to allow emission line ratio retrieval, as applied to the OI $130.4 \mathrm{~nm}$ and $135.6 \mathrm{~nm}$ emissions at Ganymede. The ratio of these emissions provides information about the atmospheric composition, specifically the relative abundances of $\mathrm{O}$ and $\mathrm{O}_{2}$. Using modelled FUV spectra representative of Ganymede's atmosphere, based on observations by the Hubble Space Telescope (HST) Space Telescope Imaging Spectrograph (STIS), we find that the accuracy of the retrieved ratios is a function of the magnitude of the ratio, with the best measurements corresponding to a ratio of $\sim 1.3$.
\end{abstract}

Keywords: FUV Imaging, FUV spectroscopy, Ganymede, aurora, atmospheres

\footnotetext{
${ }^{*}$ Corresponding author at: Department of Physics and Astronomy, University of Leicester, University Road, Leicester, UK. Tel: +44 1162525850

Email address: pmm13@leicester.ac.uk (P. M. Molyneux)
} 


\section{Introduction}

The far ultraviolet (FUV) spectral region is commonly used for auroral imaging, since several important atmospheric species, including atomic oxygen and molecular nitrogen, produce prominent FUV emissions, and the low solar flux and atmospheric reflectivity in this band result in a greatly reduced scattered sunlight background compared to that at visible wavelengths. At Earth, the brightest useful FUV auroral emissions are two oxygen multiplets - the optically allowed OI $\left({ }^{3} \mathrm{~S}^{\circ}-{ }^{3} \mathrm{P}\right)$ triplet at $130.4 \mathrm{~nm}$ and the semi-forbidden OI $\left({ }^{5} \mathrm{~S}^{\circ}{ }^{3} \mathrm{P}\right)$ doublet at $135.6 \mathrm{~nm}$ - and the Lyman-Birge-Hopfield (LBH) bands of $\mathrm{N}_{2}$, which are often considered to consist of two spectral regions: LBH-short $(140-160 \mathrm{~nm})$ and LBH-long $(160-180 \mathrm{~nm})$. Since each of these emissions is caused by electron impact excitation, isolation of the emissions allows characterisation of the flux and energy spectrum of the responsible precipitating electrons, which are useful diagnostics of magnetospheric dynamics. For example, LBH-short emissions are absorbed by the Schumann-Runge continuum of $\mathrm{O}_{2}$, while LBH-long emissions are not. As the concentration of atmospheric $\mathrm{O}_{2}$ is a function of altitude, the ratio of LBH-short to LBH-long intensity allows the determination of the depth of penetration, and hence energy, of the impinging electrons (e.g. Germany et al. 1990).

Here, we describe work to show that useful spectral information may be extracted from auroral images obtained by a compact broadband FUV imager by the introduction of two simple, commercially available transmission filters to the optical design. The study described was performed during the development of an imager proposed for ESA's JUpiter ICy moons Explorer (JUICE) mission to Jupiter, but the instrument design is also suited to observations of other FUV-emitting objects, including the Earth, and its low mass and volume should make it particularly attractive for small satellite missions.

Previous attempts at spectral isolation by auroral imagers have made use of various methods, with mixed results. The Viking and Freja satellites each carried two compact Cassegrain cameras with different broadband filters covering 134-180 nm and 123.5-160 nm (125-160 nm for Freja) to allow some wavelength selection (Anger et al. 1987; Murphree et al. 1994). The throughput of one camera was thus dominated by LBH-long emissions, while the other was mainly sensitive to the $130.4 \mathrm{~nm}$ atomic oxygen line. This limited the accuracy of any electron information derived from the ratio of emission intensities measured by the two cameras, since the relative intensities of the $\mathrm{N}_{2}$ and $\mathrm{O}$ emissions are affected by the $\mathrm{N}_{2} / \mathrm{O}$ ratio in the atmosphere, which is not constant but varies with altitude and magnetospheric activity (e.g. Mayr and Volland 1972; 
Hays et al. 1973). Better wavelength selection was achieved by the Polar UVI instrument using multilayer filters, which consist of alternating layers of high- and low-refractive index materials with carefully selected optical thicknesses such that light reflected from the boundaries between the layers experiences constructive interference at the wavelength of interest and destructive interference at other wavelengths. The Polar UVI oxygen filters each had $5 \mathrm{~nm}$ wide bandpasses and average out-of-waveband blocking of better than $3 \times 10^{-3} \%$ (Zukic et al. 1993), but the filters required a $45^{\circ}$ angle of incidence, increasing instrument complexity - the Polar UVI mass of 21 $\mathrm{kg}$ was substantially larger than the $7 \mathrm{~kg}(9.1 \mathrm{~kg})$ total mass for the Viking (Freja) cameras (Torr et al. 1995; Anger et al. 1987; Murphree et al. 1994). Good FUV wavelength discrimination may also be achieved with the use of imaging spectrometers, such as the Spectrographic Imager (SI) on IMAGE (Mende et al. 2000; IMAGE also carried a Wideband Imaging Camera (WIC) based on the Viking/Freja Cassegrain design) but, as with the Polar UVI filters, this comes at the cost of increased instrument complexity and reduced throughput. Typically, an imaging spectrograph will have a narrow field of view and must therefore build up auroral images by scanning over the auroral region, and so is unable to provide an instantaneous view of auroral morphology in the same way that a conventional imager can. Such large-scale, instantaneous images are vital for an understanding of short-timescale magnetospheric dynamics.

To clarify the purpose and advantage of our proposed filter design, we summarise the key elements of the instrument for which it was originally designed, before considering its applications to other mission opportunities.

\section{Basic instrument overview}

The Jupiter system Ultraviolet Dynamics Experiment (JUDE) was a wide-field UV imager proposed for the JUICE mission by an international consortium led by the Université de Liège and the University of Leicester. A schematic showing the JUDE optical layout is given in Figure 1. The instrument was designed to accommodate a large dynamic range, responding to both the most intense ( mega Rayleigh (Gérard et al. 1994; Ballester et al. 1996; Prangé et al. 1998; Waite Jr. et al. 2001)) emissions from Jupiter's FUV aurora and the weak ( 10s of Rayleigh (Hall et al. 1995; 1998; Roesler et al. 1999; Feldman et al. 2000; Strobel et al. 2002)) emissions from the atmospheres of the Galilean moons. A $6^{\circ}$ instrument field of view would allow the full disc of Jupiter to be imaged from a distance of $\sim 9.5 \mathrm{R}_{J}$ - the closest approach made by JUICE in the Jupiter orbit phases of the mission. Full-disc imaging of Ganymede, Callisto and Europa would also have been possible during flybys of the moons. The spatial resolution of 
the imager was designed such that when viewing Jupiter's aurora from Ganymede orbit, the resolution would have improved on the best Hubble Space Telescope (HST) images of the Jovian aurora. STIS FUV images have an angular resolution limited by the FUV-MAMA detector, which has a point spread function of $\sim 0.1$ " at $143 \mathrm{~nm}$ (Walsh 1997). At the closest possible Earth-Jupiter distance of $\sim 3.95 \mathrm{AU}$, the best spatial resolution achievable by the STIS FUVMAMA is therefore $\sim 286 \mathrm{~km}$. JUDE's angular resolution of 20 arcseconds corresponds to a spatial resolution of $\sim 100 \mathrm{~km}$ when observing Jupiter's aurora from orbit around Ganymede, a distance of $\sim 15 \mathrm{R}_{J}$ from Jupiter. In addition to this improved spatial resolution, the use of an imager rather than a scanning instrument would have provided an inherently high temporal resolution. The combination of the wide field of view, large dynamic range and high spatial and temporal resolution would allow the FUV emissions from Jupiter and its moons to be more comprehensively studied than previously possible, with unprecedented resolution and coverage. When combined with in situ particle and magnetic field data from other JUICE instruments, the JUDE auroral images would have provided new insights into the dynamic processes within Jupiter's magnetosphere and the magnetosphere-ionosphere coupling there.

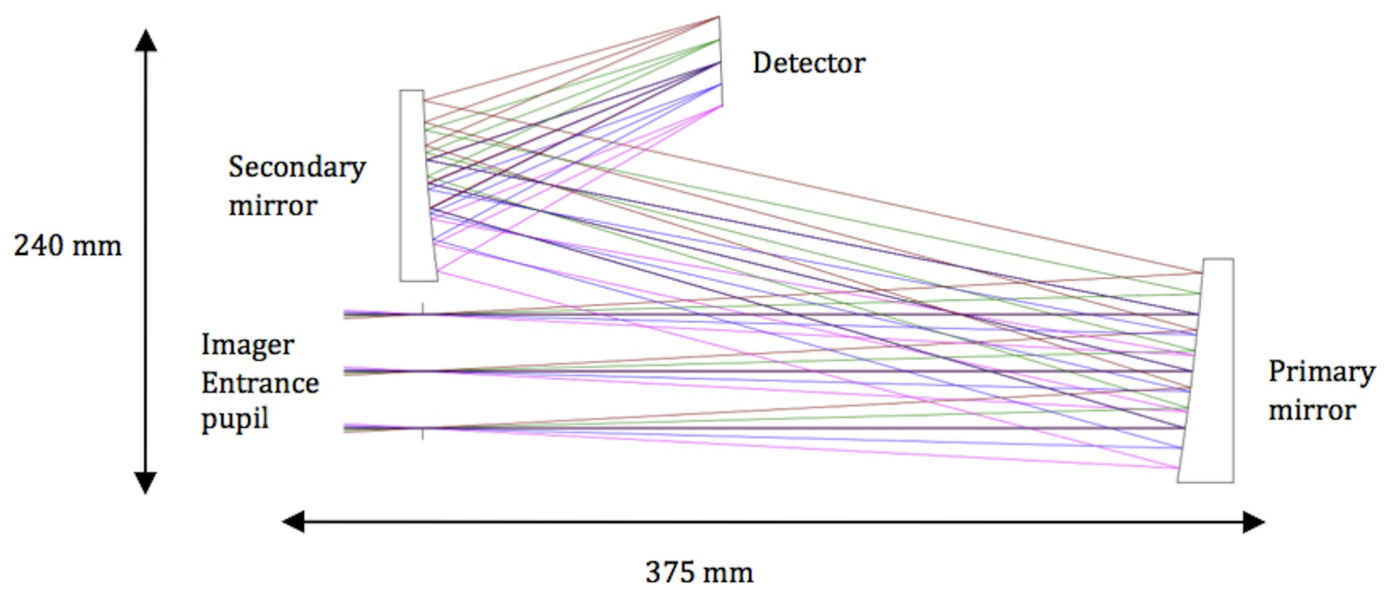

Figure 1: Schematic showing the basic optical layout proposed for the JUDE imager.

Although JUDE was not selected for JUICE, its compact, simple normal incidence design makes it an attractive option for other planetary missions or small Earth-observation satellites, particularly if some spectral capabilities can be added without a corresponding increase in the instrument complexity. The possibility of adding a spectral discrimination method was investigated for JUDE, with the aim of increasing the instrument science yield by allowing some 
characterisation of the atmospheres of the Galilean moons.

JUDE's optics consist of nickel-plated aluminium mirrors, onto which multilayer reflective coatings are deposited. The instrument bandpass is $122-165 \mathrm{~nm}$, with the mirror coatings designed to maximise the throughput around 130-143 nm (the mirror response is shown in Figure 2). Placing two or more normal-incidence transmission filters immediately in front of the focal plane detector allows multiple bandpasses to be defined within this range. Since JUICE will end its mission in orbit around Ganymede, the possibility of isolating the two main FUV emissions found here, the OI multiplets at $130.4 \mathrm{~nm}$ and $135.6 \mathrm{~nm}$, was considered. For the purpose of the study, two filters were chosen, with peak transmissions at $122 \mathrm{~nm}$ and $147 \mathrm{~nm}$ : their transmission curves are shown in Figure 2. Although these normal-incidence filters are broadband devices with overlapping bandpasses, the resulting instrument throughput in the 122 $\mathrm{nm}$ channel is higher for the $130.4 \mathrm{~nm}$ OI multiplet, while the $147 \mathrm{~nm}$ channel is biased towards detection of the $135.6 \mathrm{~nm}$ OI multiplet. Hence, if no other spectral feature than these two multiplets is present, the ratio of the intensities of the two emissions can be recovered from images obtained by the instrument. This condition is met for the nightside of Ganymede, where the only expected FUV emissions are the two OI multiplets and Lyman- $\alpha$, which the JUDE bandpass was specifically designed to exclude. On Ganymede's dayside, the instrument would detect an additional reflected solar Carbon emission at $133.5 \mathrm{~nm}$, which would make the ratio retrieval slightly more complicated. The case of dayside observations is considered briefly in Section 4.
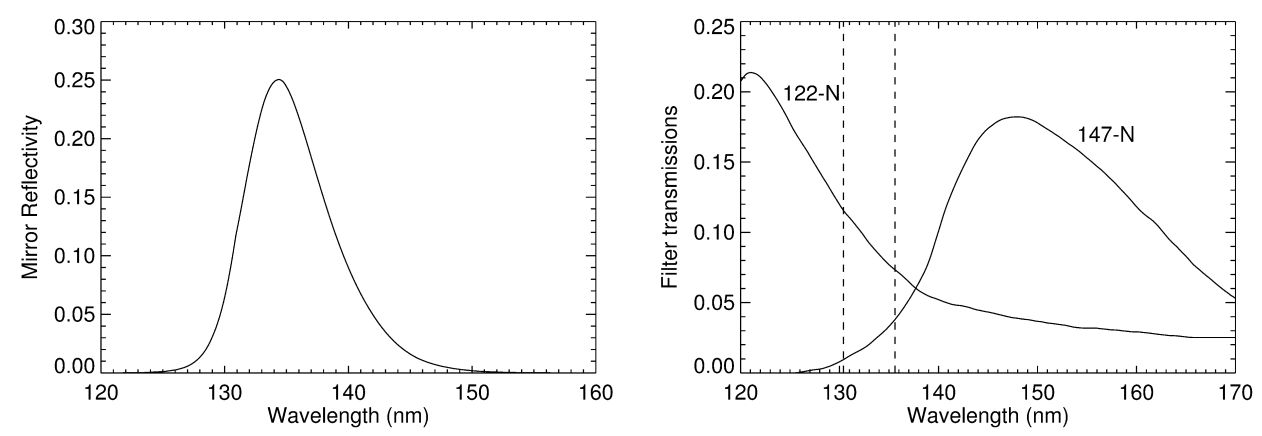

Figure 2: Left: Reflectivity of the JUDE mirror coating. Right: Transmissions of the two filters considered for JUDE, over the range of wavelengths the instrument is designed to respond to. The vertical dashed lines indicate the wavelengths of the multiplets OI seen at Ganymede. The transmission curves are based on filters $122-\mathrm{N}$ and 147-N by Acton Optics (http://www.princetoninstruments.com/products/optics/filters/curves.aspx; last accessed 17 July 2014). 


\section{Overview of Ganymede's FUV emissions}

Ganymede's oxygen atmosphere was discovered in HST Goddard High Resolution Spectrograph (GHRS) observations, which revealed FUV emissions from the OI multiplets at 130.4 $\mathrm{nm}$ and $135.6 \mathrm{~nm}$ on the satellite's trailing hemisphere (Hall et al. 1998). The double-peaked structure of the $135.6 \mathrm{~nm}$ multiplet measured in the GHRS campaign implied that the emissions had a non-uniform spatial distribution, concentrated in two distinct polar cap regions. This distribution was later confirmed by an HST Space Telescope Imaging Spectrograph (STIS) observation campaign, in which Ganymede's trailing hemisphere was observed over four HST orbits (Feldman et al. 2000). Examples of the STIS and GHRS measurements performed during these campaigns are shown in Figure 3. Field and particle measurements performed during Galileo flybys of Ganymede had previously shown that the moon possesses an internal magnetic field strong enough to generate a miniature magnetosphere within Jupiter's magnetosphere (Gurnett et al. 1996; Kivelson et al. 1996; 1997; 1998; Williams et al. 1997). Analysis of the STIS Ganymede images revealed that the brightest emissions occur at the boundaries of the polar caps, where the open/closed field line boundary of the magnetic field intersects the atmosphere (Feldman et al. 2000), implying that the emissions are of an auroral nature. Significant variability in the location and intensity of emissions, and in the relative intensity of the northern and southern hemisphere emissions, was seen between images obtained from different HST orbits. Feldman et al. ascribed the variations to the tilted jovian field leading to changes in the location of Ganymede's polar caps and open/closed field line boundary regions over a jovian rotation.
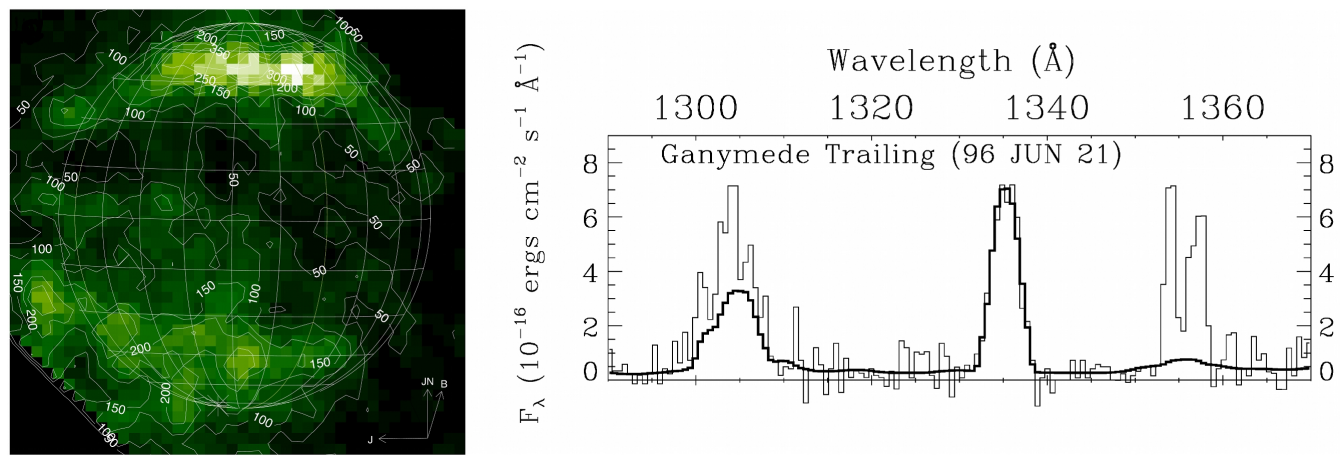

Figure 3: Left: STIS image of Ganymede's $135.6 \mathrm{~nm}$ emission from Feldman et al. (2000). Right: GHRS FUV spectrum of Ganymede extracted from Figure 1 of Hall et al. (1998). The thick black line indicates spectral contributions from reflected sunlight.

To date, the only published FUV images of Ganymede's aurora and airglow are the Feldman 
STIS observations and composite images from three further HST campaigns (two STIS, one using the Advanced Camera for Surveys) shown in McGrath et al. (2013). While the four HST campaigns provided images of Ganymede's leading and trailing hemispheres, and the intermediate Jupiter-facing hemisphere, the images at each position were obtained at intervals of several years. The brightest FUV emissions at Ganymede are auroral, and therefore indicative of the plasma conditions in Ganymede's magnetosphere, which vary on both short (minutes to hours) and long (years) timescales, as evidenced by changes in the intensity and location of the Ganymede footprint aurora on Jupiter (Grodent et al. 2008; 2009). McGrath et al. show that the position of the auroral ovals appears to be stable over long periods, but there are significant brightness fluctuations between individual images of the emissions. Hence, further images showing the variability of the emissions on various timescales (for example: one full jovian rotation, one solar rotation, Ganymede's orbital period), will enhance our understanding of the interaction between the magnetospheres of Jupiter and Ganymede. Additionally, a knowledge of the relative intensities of the $130.4 \mathrm{~nm}$ and $135.6 \mathrm{~nm}$ emissions will provide information on Ganymede's atmospheric composition by allowing the relative abundances of $\mathrm{O}$ and $\mathrm{O}_{2}$ to be constrained. Since the most probable mechanisms driving the OI emissions are believed to be electron-impact excitation of $\mathrm{O}$ atoms and electron-impact dissociative excitation of $\mathrm{O}_{2}$ (Hall et al. 1998; Feldman et al. 2000), the magnitude of the emission ratio is dependent on the energy of the electrons as well as the atmospheric composition. In a pure atomic oxygen atmosphere, an intensity ratio $135.6 \mathrm{~nm}: 130.4 \mathrm{~nm}$ of less than 1.2:1 is expected at Ganymede (Feldman et al. 2000), while the ratio during the STIS imaging campaign was found to vary between $1.2 \pm 0.2$ and $3.2 \pm 1.6$ (Feldman et al. 2000), implying that the atmosphere is dominated by $\mathrm{O}_{2}$. A thorough imaging campaign combining large-scale images of the emission regions with information about spatial and temporal variations in the OI FUV emission ratio would allow models of Ganymede's atmospheric composition and auroral production mechanisms to be refined. An in situ imager such as JUDE is ideal for this purpose, as the increased throughput relative to STIS would allow instantaneous imaging with improved spatial resolution, and images may be compared to simultaneous field and particle measurements obtained from the same platform.

\section{Ratio calculation method}

We assume no significant FUV emission from Ganymede's nightside atmosphere in the JUDE bandpass other than the two OI emissions, based on the low background seen in the HST/GHRS observation of Ganymede (Hall et al. (1998) and Figure 3 above). The total count rate, $C_{\text {total }}$, 
through each of the two transmission filters is then given by

$$
\begin{aligned}
& C_{\text {total }(a)} \propto t_{130.4(a)} r_{130.4} F_{130.4}+t_{135.6(a)} r_{135.6} F_{135.6} \\
& C_{\text {total }(b)} \propto t_{130.4(b)} r_{130.4} F_{130.4}+t_{135.6(b)} r_{135.6} F_{135.6}
\end{aligned}
$$

where $r$ is the reflectivity of the JUDE mirror coatings at the specified wavelength, $F$ is the emitted atmospheric flux, and $t$ is the transmission of the relevant filter, where $(a)$ and $(b)$ represent the $122 \mathrm{~nm}$ and $147 \mathrm{~nm}$ filters respectively. (To convert these proportionalities to full equations, additional terms such as the instrument aperture and detector quantum efficiency are required. For now we assume that these values are constant over the $130-136 \mathrm{~nm}$ region, so while the calculated emitted flux values will differ from the true value at Ganymede, the ratio of the fluxes at the two wavelengths of interest will be correct.) The transmission and reflectivity values used in the calculation were averages taken over $130.1-130.7 \mathrm{~nm}$ for the $130.4 \mathrm{~nm}$ emissions and $135.5-135.9 \mathrm{~nm}$ for the $135.6 \mathrm{~nm}$ emissions, since both emissions are multiplets. The equations can be solved to give the raw emitted fluxes at the two wavelengths of interest:

$$
\begin{gathered}
F_{130.4} \propto \frac{C_{\text {total }(b)}-\frac{t_{135.6(b)} C_{\text {total }(a)}}{t_{135.6(a)}}}{t_{130.4(b)} r_{130.4}-\frac{t_{135.6(b)} t_{130.4(a)} r_{130.4}}{t_{135.6(a)}}}, \\
F_{135.6} \propto \frac{C_{\text {total }(a)}-t_{130.4(a)} r_{130.4} F_{130.4}}{t_{135.6(a)} r_{135.6}} .
\end{gathered}
$$

If this method were used to study Ganymede's dayside emissions, a correction for reflected sunlight would be required. An appropriate solar spectrum as obtained, for example, by the SOLSTICE instrument on the SORCE satellite (McClintock et al. 2005) could be used along with a measure of Ganymede's UV albedo to estimate the count rate through each filter due to reflected sunlight. The solar component may then be subtracted from the total counts through each filter before the ratio calculation is performed. For this initial study, however, we consider the simpler, nightside emission ratio retrieval only.

\section{Ganymede atmospheric model}

In order to test the ratio recovery technique, spectra representative of the emissions expected at Ganymede were required. The most detailed FUV spectra currently available were obtained by HST/STIS, but in these observations the Ganymede emissions are convolved with the STIS instrument response and slit width. To provide an estimate of the emitted spectra expected at 
Ganymede, the count rates contained in the STIS spectra were distributed over a basic model of Ganymede's atmosphere.

The model was produced with the assumption that the only emissions within the $128 \mathrm{~nm}$ to $138 \mathrm{~nm}$ region on Ganymede's nightside are the OI emissions at $130.4 \mathrm{~nm}$ and $135.6 \mathrm{~nm}$. This assumption was based on the HST/GHRS spectrum of Ganymede's trailing hemisphere shown in Hall et al. (1998) and reproduced in Figure 3, which covers the same region and shows no features that cannot be attributed to reflected sunlight other than the oxygen lines. The OI emission region around $130.4 \mathrm{~nm}$ is a triplet with components at $130.217 \mathrm{~nm}, 130.486 \mathrm{~nm}$ and $130.603 \mathrm{~nm}$, while the $135.6 \mathrm{~nm}$ emissions consist of a doublet at $135.560 \mathrm{~nm}$ and $135.851 \mathrm{~nm}$ (Wiese et al. 1996). The finite width of these lines is a consequence of Doppler broadening and is governed by the temperature of the oxygen responsible for the emissions. However, little is known about Ganymede's atmosphere, including its temperature, so the energy of the excited $\mathrm{O}$ atoms is not easily characterised. Our approach was therefore to consider two line width estimates, representing thermodynamic equilibrium and non-equilibrium conditions in the $\mathrm{O}$ atom population.

The first estimate assumes thermodynamic equilibrium conditions with a temperature of 600 K. Modelling by Marconi (2007) shows that the temperature of atmospheric species at Ganymede is dependent on altitude and subsolar latitude. A temperature of $600 \mathrm{~K}$ falls within the expected range of temperatures for oxygen atoms with altitudes up to $\sim 200 \mathrm{~km}$. The FWHM of the broadened lines is then given by

$$
\Delta \lambda_{F W H M}=\sqrt{\frac{8 k T \ln 2}{m c^{2}}} \lambda_{0},
$$

where $k$ is the Boltzmann constant, $T$ is the temperature, $m$ is the mass of an oxygen atom, $c$ is the speed of light in vacuum and $\lambda_{0}$ is the central wavelength of the emission line.

Since the line widths calculated using Equation 5 are valid for the case where the $\mathrm{O}$ atoms are in thermodynamical equilibrium, their velocity distribution is Maxwellian. However, the process leading to the excitation of the $130.4 \mathrm{~nm}$ and $135.6 \mathrm{~nm}$ oxygen multiplets is believed to be dissociative excitation of $\mathrm{O}_{2}$ by energetic electrons, and consequently, it is possible that the excited $\mathrm{O}$ atoms are not in equilibrium, with the kinetic energy of the atoms dependent on the energy of the impacting electrons. Studies of dissociative excitation of $\mathrm{O}_{2}$ have shown that at electron impact energies of $20 \mathrm{eV}$, the resulting OI energy distribution is narrow, with a peak around $0.13 \mathrm{eV}$ and no fragments with energies $>2 \mathrm{eV}$ observed (Mason and Newell 1990). As the electron energy is increased, the distribution broadens and at $100 \mathrm{eV}$ the OI energy has a 
peak at $1.3 \pm 0.5 \mathrm{eV}$, with FWHM $3.7 \pm 1.4 \mathrm{eV}$ and mean of $2.4 \pm 1.0 \mathrm{eV}$ (Makarov et al. 2003). A second line width estimate was therefore made, assuming that the two $\mathrm{O}$ atoms produced by the excitation of each $\mathrm{O}_{2}$ molecule are not in equilibrium. The jovian magnetospheric electron temperature at Ganymede orbit is not well known - Feldman et al. consider a range of 1-100 $\mathrm{eV}$ in their analysis, and Eviatar et al. (2001) calculate that an electron population characterised by a Maxwellian distribution with temperatures in the range $75-300 \mathrm{eV}$ is required to excite the brightest auroral emissions - so an estimate of $5 \mathrm{eV}$ for the kinetic energy of the dissociation fragments was considered. This value falls within the kinetic energy distributions plotted by Makarov et al. for electron temperatures of $60 \mathrm{eV}$ and upwards. The estimated energy of the $\mathrm{O}$ atoms was converted into a velocity using the standard kinetic energy formula $E=\frac{1}{2} m v^{2}$. An upper limit for the line width can then be calculated by assuming that the $\mathrm{O}$ atoms travel in opposite directions with their velocities directly toward or away from the observer. The Doppler shift in each multiplet component emission wavelength in each case can be calculated from

$$
\Delta \lambda=\frac{v}{c} \lambda_{0},
$$

and the maximum line width is the difference between the two Doppler-shifted emission wavelengths. The line widths calculated for each OI multiplet using each of the two approximations are given in Table 1 . The non-equilibrium line widths are a factor of $\sim 6$ larger than the equivalent lines in the thermodynamic equilibrium case, but in either case the widths are very small in comparison to the wavelength difference between the two multiplets.

Table 1: Estimated line widths for O I multiplet emissions at Ganymede

\begin{tabular}{lll}
\hline \hline & \multicolumn{2}{c}{ Line widths $(\mathrm{nm})$} \\
Wavelength $(\mathrm{nm})$ & $\begin{array}{l}\text { Thermodynamic } \\
\text { equilibrium case }\end{array}$ & \multicolumn{1}{c}{ Non-equilibrium } \\
& & case \\
& & \\
\hline 130.217 & $5.71 \times 10^{-4}$ & $3.37 \times 10^{-3}$ \\
130.486 & $5.72 \times 10^{-4}$ & $3.38 \times 10^{-3}$ \\
130.603 & $5.72 \times 10^{-4}$ & $3.38 \times 10^{-3}$ \\
& & \\
135.560 & $5.94 \times 10^{-4}$ & $3.51 \times 10^{-3}$ \\
135.851 & $5.95 \times 10^{-4}$ & $3.52 \times 10^{-3}$ \\
\hline \hline
\end{tabular}


In the approximation that Ganymede's atmosphere is optically thin, the intensities of the multiplet component lines are in the ratio of their transition probabilities, which are given in Table 2 (values taken from Wiese et al. 1996). To create the Ganymede FUV spectral model, data were extracted from HST/STIS observations of Ganymede and the count rates for the regions around $130.4 \mathrm{~nm}$ and $135.6 \mathrm{~nm}$ calculated and redistributed to the constituent multiplet lines according to the ratios in Table 2. The number of counts in each line was then distributed into a Gaussian, initially with a FWHM as calculated for $\mathrm{O}$ atoms in thermodynamic equilibrium at $600 \mathrm{~K}$ (Table 1, middle column), and then in a separate procedure using the line width calculated for $\mathrm{O}$ atoms that are not in equilibrium (Table 1, right column). In this way, two spectra were produced for each of the STIS images, to allow the accuracy of the $130.4 \mathrm{~nm}: 135.6 \mathrm{~nm}$ calculation to be investigated for two different atmospheric approximations.

Table 2: Transition probabilities for OI emissions around $130.4 \mathrm{~nm}$ and $135.6 \mathrm{~nm}$ (Wiese et al. 1996).

\begin{tabular}{lc}
\hline \hline & \\
Wavelength $(\mathrm{nm})$ & Transition probability $\left(\mathrm{s}^{-1}\right)$ \\
& \\
\hline 130.217 & $3.41 \times 10^{8}$ \\
130.486 & $2.03 \times 10^{8}$ \\
130.603 & $6.76 \times 10^{7}$ \\
& \\
135.560 & $4.20 \times 10^{3}$ \\
135.851 & $1.36 \times 10^{3}$ \\
\hline \hline
\end{tabular}

The count rate information required for the atmospheric model was extracted from the eight STIS observations of Ganymede's trailing hemisphere described in Feldman et al. (2000) (image IDs o53k01010 - o53k01080, obtained over four contiguous HST orbits on October 30th, 1998). Spectra were produced from the "x2d" data files, calibrated 2D spectral image files in which the signal is given in units of $\mathrm{erg} \mathrm{s}^{-1} \mathrm{~cm}^{-2} \AA^{-1} \operatorname{arcsec}^{-1}$, following the method described in Feldman et al. (2000). An appropriate solar spectrum from UARS SOLSTICE (Rottman et al. 1993) was fitted to the STIS spectra to allow correction for reflected sunlight, and spectra extracted from the STIS images in regions away from the position of Ganymede in the slit were used to correct for background including light scattered by the geocorona.

In order to determine the flux due to OI emissions in the extracted spectra, the spatial 
distribution of the emissions had to be considered. The slit used for the STIS observations had a width of 2 arcsec, which corresponds to $\sim 82$ pixels. The G140L grating used to obtain the STIS images has a dispersion of $0.0584 \mathrm{~nm}$ pixel $^{-1}$, which means that emissions at any specific wavelength which are spatially distributed over the width of the slit will appear to be spread over $\sim 4.8 \mathrm{~nm}$. Hence, emissions at $130.4 \mathrm{~nm}$ may appear anywhere between 128.0 and $132.8 \mathrm{~nm}$ in the STIS spectra, and emissions at $135.6 \mathrm{~nm}$ may appear between 133.2 and $138.0 \mathrm{~nm}$. Since these emission regions do not overlap, and no other FUV emissions were expected in the 128.0$138.0 \mathrm{~nm}$ part of the spectra, the $130.4 \mathrm{~nm}$ flux for each of the extracted spectra was calculated by summing over $128.0-132.8 \mathrm{~nm}$ and the $135.6 \mathrm{~nm}$ flux by summing over $133.2-138.0 \mathrm{~nm}$. The two flux values were then distributed into the constituent multiplet emission lines, according to the ratios of their transmission probabilities (Table 2), to produce models of the raw Ganymede emission spectra at the time of each STIS observation.

In order to create more representative spectra, a noise contribution was then added. Even if it is correct to assume that the only emissions within the JUDE bandpass at Ganymede are the $130.4 \mathrm{~nm}$ and $135.6 \mathrm{~nm}$ lines, and assuming no stray light contamination and no electronic noise, the JUDE detector would still record counts due to the high jovian radiation background. Since JUDE made use of an MCP detector, with no intrinsic energy resolution, this noise contribution was modelled as a fixed offset, with the number of noise counts per spectral bin simply equal to the total noise counts divided by the number of bins. Poisson statistics were then applied to make the noise distribution more realistic. A typical MCP detector has an intrinsic noise level of $\sim 0.1$ counts $\mathrm{cm}^{-2} \mathrm{~s}^{-1}$, but at Ganymede JUDE would also detect noise counts from the intense charged particle background of Jupiter's magnetosphere. Modelling of the radiation dose expected at the JUDE detector in such an environment has not been performed in detail, so for the purpose of the current work three noise levels were investigated, based on multiples of the signal count rates, so that signal : noise levels of $5: 1,2: 1$ and $1: 1$ were explored.

\section{Ratio recovery tests}

The count rate in each wavelength bin of a modelled Ganymede spectrum was multiplied by the combined throughput of the JUDE reflective optics and one of the FUV transmission filters at the relevant wavelength, resulting in two spectra each showing the final count rate after focusing by the instrument and transmission through one of the two filters. The counts in each spectrum were then summed to give the quantities $C_{\text {total(a) }}$ and $C_{\text {total(b) }}$ required by Equations 3 and 4 . These equations were then solved, using the known filter transmissions and coating 
reflectivity for the $t$ and $r$ terms, to give the raw emitted flux in the $130.4 \mathrm{~nm}$ and $135.6 \mathrm{~nm}$ multiplets of the model atmosphere. This detected flux was compared to the incident flux shown in the initial model spectrum to give an indication of how well the ratio of the two emissions could be recovered through the calculation method.

The results of the ratio recovery calculations for the Ganymede spectral models described above are given in Table 3. The results for an atmosphere in thermodynamic equilibrium and for the non-equilibrium case were very similar, and the calculated percentage differences between the incident emission ratio and the detected emission ratio for the two models differed by at most $2 \times 10^{-3}$ percentage points. For this reason, only the results of the non-equilibrium model, with line widths of $\sim 3.4 \times 10^{-3} \mathrm{~nm}$, are included in the tables. It should be noted that the 135.6 $\mathrm{nm}: 130.4 \mathrm{~nm}$ emission ratios derived from the model spectra are in most cases different to the ratios given by Feldman et al. for the same STIS observations (see Table 1 in Feldman et al. 2000). This may be due to slight differences in the data extraction methods used, particularly where the solar spectrum was fitted to the Ganymede spectrum. A spectrum extracted from image o53k01060 is shown in Figure 4 for comparison with Figure 2 in Feldman et al. (2000), which shows a spectrum extracted from the same image. While the agreement is generally good, small differences in the shapes of the OI lines can be seen. However, the range of ratios observed is similar $(\sim 1.2 \pm 0.4-2.7 \pm 1.8$ in this work compared to $1.2 \pm 0.3-3.2 \pm 1.6$ in Feldman et al. 2000; all errors are calculated from $1 \sigma$ statistical uncertainty in the observed emission fluxes) so the spectra are still representative of emissions observed at Ganymede

As expected, an increase in the noise level of the spectral models leads to generally larger discrepancies between the incident and detected ratios. The magnitude of the difference appears to be a function of the ratio itself, with a minimum at a ratio of $\sim 1.3$ (image o53k01060). The shape of this function is shown in Figure 5. The calculated ratio for images o53k01050 and o53k01030 (both to the right of the minimum in Figure 5) were underestimates, while for all other images (with ratios of $\sim 1.3$ or less) the ratio was overestimated. The largest discrepancy between the detected and incident results was found for the largest ratio, $\sim 2.8$ (image o53k01030). At $\mathrm{S}: \mathrm{N}=1: 1$, the measured ratio for this image was underestimated by $\sim 30 \%$. For comparison, the ratios measured by Feldman et al. are quoted with uncertainties of between $\sim 13$ and $50 \%$ of the measured value. 

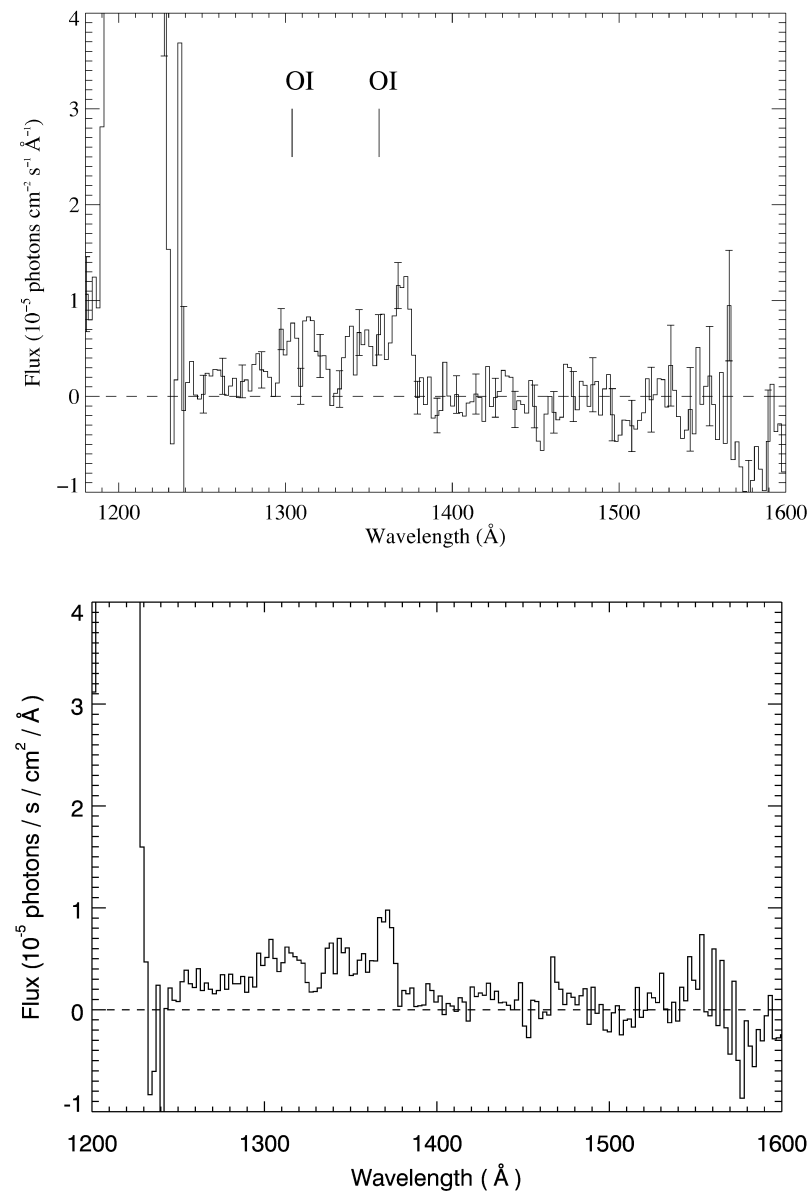

Figure 4: Comparison of two spectra obtained from the same HST/STIS image (ID o53k01060). The upper plot was created by Feldman et al. (2000), while the lower plot was extracted during the current work. Negative flux values stem from the estimated reflected sunlight component that was removed from the raw spectrum. 
Table 3: Results of emission ratio calculation for spectral models based on STIS observations of Ganymede with various $\mathrm{S}: \mathrm{N}$ levels

\begin{tabular}{|c|c|c|c|c|c|c|c|}
\hline \multirow[t]{2}{*}{ Image ID } & \multirow[t]{2}{*}{ Incident ratio } & \multicolumn{2}{|c|}{$\mathrm{S}: \mathrm{N}=5: 1$} & \multicolumn{2}{|c|}{$\mathrm{S}: \mathrm{N}=2: 1$} & \multicolumn{2}{|c|}{$\mathrm{S}: \mathrm{N}=1: 1$} \\
\hline & & $\begin{array}{l}\text { Detected } \\
\text { ratio }\end{array}$ & $\begin{array}{l}\text { Difference } \\
(\%)\end{array}$ & $\begin{array}{l}\text { Detected } \\
\text { ratio }\end{array}$ & $\begin{array}{l}\text { Difference } \\
(\%)\end{array}$ & $\begin{array}{l}\text { Detected } \\
\text { ratio }\end{array}$ & $\begin{array}{l}\text { Difference } \\
(\%)\end{array}$ \\
\hline o53k01010 & 1.2018 & 1.2160 & 1.182 & 1.2435 & 3.470 & 1.2726 & 5.891 \\
\hline o53k01020 & 1.2589 & 1.2836 & 1.962 & 1.2995 & 3.225 & 1.3159 & 4.528 \\
\hline o53k01030 & 2.7905 & 2.4779 & 11.202 & 2.1983 & 21.222 & 1.9531 & 30.009 \\
\hline o53k01040 & 1.2546 & 1.2819 & 2.176 & 1.2980 & 3.459 & 1.3148 & 4.798 \\
\hline o53k01050 & 1.6213 & 1.5874 & 2.091 & 1.5435 & 4.799 & 1.4999 & 7.488 \\
\hline o53k01060 & 1.3230 & 1.3481 & 1.897 & 1.3522 & 2.207 & 1.3565 & 2.532 \\
\hline o53k01070 & 1.2137 & 1.2486 & 2.876 & 1.2706 & 4.688 & 1.2936 & 6.583 \\
\hline o53k01080 & 1.2098 & 1.2436 & 2.794 & 1.2664 & 4.678 & 1.2904 & 6.662 \\
\hline
\end{tabular}

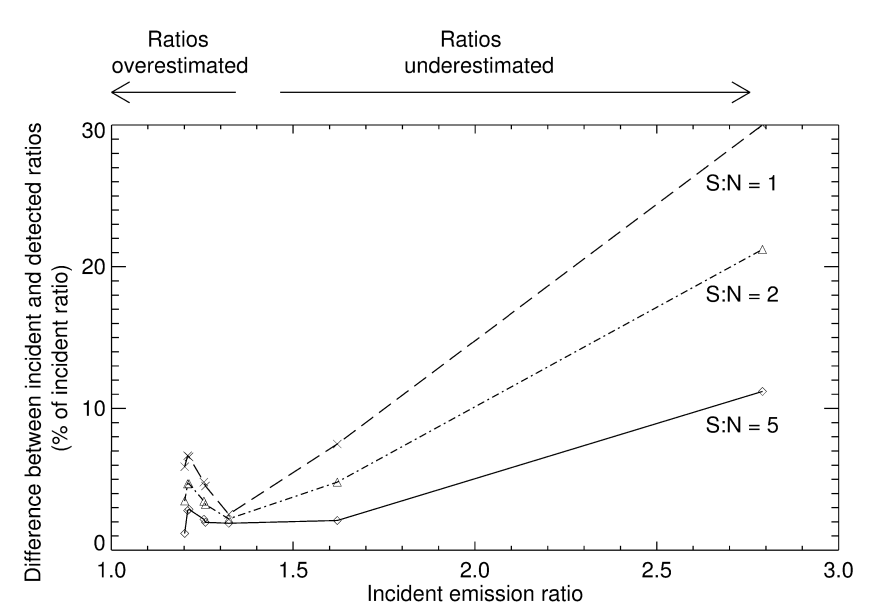

Figure 5: Relationship between the magnitude of the 135.6 : $130.4 \mathrm{~nm}$ intensity ratio and the uncertainty associated with the measurement of the ratio, for a range of $\mathrm{S}: \mathrm{N}$ values. 


\section{Discussion}

The work described above shows that it is possible to retrieve spectral emission ratios from broadband instruments using a suitable combination of transmission filters. This technique allows atmospheric compositional information to be obtained simultaneously with images of atmospheric emission variability, leading to better characterisation of the emissions and the processes responsible for them. However, in a noisy environment the retrieval of accurate emission ratio information using this broadband technique is complicated by the relationship between the magnitude of the ratio and the magnitude of uncertainty expected, and this relationship should be investigated further to explore whether it is possible to predict and correct for it. The retrieval of emission ratios requires a reliable knowledge of the optical properties of the instrument, and in environments such as Jupiter's magnetosphere, where the charged particle background is high, effects such as optical darkening of the broadband filters and charging of mirror coatings may need to be corrected for.

Although the work presented here chose Ganymede as a case study, the ratio retrieval method can also be applied to emissions at other bodies, including other moons of the outer planets and the Earth. The design of instrumentation for both outer planet missions and small satellite Earth orbiting missions is driven by a need to minimise mass and volume requirements and instrument complexity, and a filtering technique which is compatible with the design of compact imagers is attractive in either situation. The normal-incidence design of the filter scheme we describe is also very relevant to the new generation of highly compact, low mass wide-field imager systems being developed using microchannel plate optics (e.g. Bannister et al. 2007), since the need for mechanisms or folded light paths in such instruments rapidly erodes the mass and volume advantages they offer. At Earth, radiation effects such as optical darkening pose less of a problem than at Ganymede, but the choice of filters is complicated by the overlap between the LBH and OI emissions: if the emission ratio is to be used to derive information about the precipitating electrons, the signal measured should be dominated by one atmospheric species to avoid ambiguities introduced by the varying atmospheric $\mathrm{N}_{2} / \mathrm{O}$ concentration ratio. This could be achieved by adjusting the filter transmissions and/or mirror reflectivities, for example by varying the thicknesses of the coating layers. The bandpass of the JUDE design can thus be optimised for the requirements of specific missions. The imager dimensions will also vary depending on the target of the mission it is used for. For example, in order to image the weakest emissions of interest at Earth ( $50 \mathrm{R}$, concentrated mainly in the $130.4 \mathrm{~nm}$ line) above a background of 
$8 \mathrm{R}$ per $0.1 \mathrm{~nm}$ (consistent with with a UV spectrum from Torr et al. 1995) to a significance of three sigma, a primary mirror of diameter $\sim 70 \mathrm{~mm}$ is required, slightly smaller than the $80 \mathrm{~mm}$ diameter JUDE mirror. The mirror size is currently limited by the low throughput of the 147 nm filter, so may potentially be reduced further by filter optimisation.

The atmospheres of the outer planetary moons are generally not well characterised, but FUV emissions have been detected at several. For example: the OI emission multiplets at $130.4 \mathrm{~nm}$ and $135.6 \mathrm{~nm}$ seen at both Earth and Ganymede can also be observed at Europa (Hall et al. 1995; 1998), Io (Ballester et al. 1987; Clarke et al. 1994; Roesler et al. 1999) and Enceladus (Zastrow et al. 2012); SI emissions at $129.9 \mathrm{~nm}, 138.9 \mathrm{~nm}, 142.9 \mathrm{~nm}$ and $147.9 \mathrm{~nm}$ are seen at Io (Ballester et al. 1987; Roesler et al. 1999); and $\mathrm{N}_{2}$ LBH band emissions have been observed at Titan (Broadfoot et al. 1981; Strobel and Shemansky 1982; Ajello et al. 2008; Stevens et al. 2011; Ajello et al. 2012) and are predicted to exist at Triton (Strobel et al. 1991). The Io torus also emits in the FUV region, with SII, SIII and SIV emissions dominating the spectrum (A Cassini UVIS spectrum is shown in Steffl et al. 2004). As with Ganymede, several of these objects exhibit variable emission morphologies for various reasons. At Io, the SI and OI emissions are mainly concentrated in two equatorial spots which are thought to be associated with fieldaligned currents that connect Io to Jupiter (Roesler et al. 1999). An asymmetry in Europa's OI airglow has also been seen in HST/STIS images (McGrath et al. 2004; 2009). This may be due to variations in the local plasma environment as Europa moves in and out of Jupiter's current sheet (McGrath et al. 2009), but has also been attributed to either a non-uniform distribution of reactive species in Europa's regolith, leading to a non-uniform removal of $\mathrm{O}_{2}$ from the atmosphere (Cassidy et al. 2007) or to a plume in the region of enhanced emission, caused by tidally driven shear heating (Saur et al. 2011; see also Roth et al. 2014 for a description of a plume at Europa detected using FUV STIS images). An instrument which combines instantaneous imaging with spectral measurements is ideal for characterising variable atmospheres like these. An in situ imager will encounter enhanced throughput compared to, for example, the HST instruments observing the moons from Earth orbit, allowing even faint, tenuous emissions to be studied.

\section{Acknowledgements}

The authors wish to thank the wider JUDE consortium, and the referee for their helpful comments which have improved this manuscript. PMM was supported for this work by an STFC studentship. 


\section{References}

J. M. Ajello, J. Gustin, I. Stewart, K. Larsen, L. Esposito, W. Pryor, W. McClintock, M. H. Stevens, C. P. Malone, and D. Dziczek. Titan airglow spectra from the Cassini Ultraviolet Imaging Spectrograph: FUV disk analysis. Geophys. Res. Lett.., 35, 2008.

J. M. Ajello, R. A. West, J. Gustin, K. Larsen, A. I. F. Stewart, L. W. Esposito, W. E. McClintock, G. M. Holsclaw, and E. T. Bradley. Cassini UVIS observations of Titan nightglow spectra. J. Geophys. Res., 117, 2012.

C. D. Anger, S. K. Babey, A. Lyle Broadfoot, R. G. Brown, L. L. Cogger, R. Gattinger, J. W. Haslett, R. A. King, D. J. McEwen, J. S. Murphree, E. H. Richardson, B. R. Sandel, K. Smith, and A. Vallance Jones. An ultraviolet auroral imager for the Viking spacecraft. Geophysical Research Letters, 14(4):387-390, 1987.

G. E. Ballester, H. W. Moos, P. D. Feldman, D. F. Strobel, M. E. Summers, J.-L. Bertaux, T. E. Skinner, M. C. Festou, and J. H. Lieske. Detection of neutral oxygen and sulfur emissions near Io using IUE. Astrophys. J., 319:L33-L38, 1987.

G. E. Ballester, J. T. Clarke, J. T. Trauger, W. M. Harris, K. R. Stapelfeldt, D. Crisp, R. W. Evans, E. B. Burgh, C. J. Burrows, S. Casertano, J. S. Gallagher III, R. E. Griffiths, J. J. Hester, J. G. Hoessel, J. A. Holtzman, J. E. Krist, V. Meadows, J. R. Mould, R. Sahai, P. A. Scowen, A. M. Watson, and J. A. Westphal. Time-resolved observations of Jupiter's far-ultraviolet aurora. Science, 274:409-413, 1996.

N. P. Bannister, E. J. Bunce, S. W. H. Cowley, R. Fairbend, G. W. Fraser, F. J. Hamilton, J. S. Lapington, J. E. Lees, M. Lester, S. E. Milan, J. F. Pearson, and R. Willingale. A Wide Field Auroral Imager (WFAI) for low Earth orbit missions. Ann. Geophys., 25:519-532, 2007.

A. L. Broadfoot, B. R. Sandel, D. E. Shemansky, J. B. Holberg, G. R. Smith, D. F. Strobel, J. C. McConnell, S. Kumar, D. M. Hunten, S. K. Atreya, T. M. Donahue, H. W. Moos, J. L. Bertaux, J. E. Blamont, R. B. Pomphrey, and S. Linick. Extreme ultraviolet observations from Voyager 1 encounter with Saturn. Science, 212:206-211, 1981.

T. A. Cassidy, R. E. Johnson, M. A. McGrath, M. C. Wong, and J. F. Cooper. The spatial morphology of Europa's near-surface $\mathrm{O}_{2}$ atmosphere. Icarus, 191:755-764, 2007.

J. T. Clarke, J. Ajello, J. Luhmann, N. Schneider, and I. Kanik. Hubble Space Telescope UV spectral observations of Io passing into eclipse. J. Geophys. Res., 99:8387-8402, 1994. 
A. Eviatar, D. F. Strobel, B. C. Wolven, P. D. Feldman, M. A. McGrath, and D. J. Williams. Excitation of the Ganymede ultraviolet aurora. Astrophys. J., 555:1013-1019, 2001.

P.D Feldman, M. A. McGrath, D.F. Strobel, H. W. Moos, K. D. Retherford, and B. C. Wolven. HST/STIS ultraviolet imaging of polar aurora on Ganymede. Astrophys. J., 535:1085-1090, 2000.

J. C. Gérard, D. Grodent, R. Prangé, J. H. Waite, G. R. Gladstone, V. Dols, F. Paresce, A. Storrs, L. Ben Jaffel, and K. A. Franke. A remarkable auroral event on Jupiter observed in the ultraviolet with the Hubble Space Telescope. Science, 266:1675-16708, 1994.

G. A. Germany, M. R. Torr, P. G. Richards, and D. G. Torr. The dependence of modeled OI 1356 and $\mathrm{N}_{2}$ Lyman Birge Hopfield auroral emissions on the neutral atmosphere. J. Geophys. Res., 95:7725-7733, 1990.

D. Grodent, J.-C. Gérard, A. Radioti, B. Bonfond, and A. Saglam. Jupiter's changing auroral location. J. Geophys. Res., 113, 2008.

D. Grodent, B. Bonfond, A. Radioti, J.-C. Gérard, X. Jia, J. D. Nichols, and J. T. Clarke. Auroral footprint of Ganymede. J. Geophys. Res., 114, 2009.

D. A. Gurnett, W. S. Kurth, A. Roux, S. J. Bolton, and C. F. Kennel. Evidence for a magnetosphere at Ganymede from plasma-wave observations by the Galileo spacecraft. Nature, 384: $535-537,1996$.

D. T. Hall, D. F. Strobel, P. D. Feldman, M. A. McGrath, and H. A. Weaver. Detection of an oxygen atmosphere on Jupiter's moon Europa. Nature, 373:677-679, 1995.

D. T. Hall, P. D. Feldman, M. A. McGrath, and D. F. Strobel. The far-ultraviolet oxygen airglow of Europa and Ganymede. Astrophys. J., 499:475-481, 1998.

P. B. Hays, R. A. Jones, and M. H. Rees. Auroral heating and the composition of the neutral atmosphere. Planet. Space Sci., 21:559-573, 1973.

M. G. Kivelson, K. K. Khurana, C. T. Russell, R. J. Walker, J. Warnecke, F. V. Coroniti, C. Polanskey, D. J. Southwood, and G. Schubert. Discovery of Ganymede's magnetic field by the Galileo spacecraft. Nature, 384:537-541, 1996. 
M. G. Kivelson, K. K. Khurana, F. V. Coroniti, S. Joy, C. T. Russell, R. J. Walker, J. Warnecke, L. Bennett, and C. Polanskey. The magnetic field and magnetosphere of Ganymede. Geophys. Res. Lett., 24(17):2155-2158, 1997.

M. G. Kivelson, J. Warnecke, L. Bennett, S. Joy, K. K. Khurana, J. A. Linker, C. T. Russell, R. J. Walker, and C. Polanskey. Ganymede's magnetosphere: magnetometer overview. J. Geophys. Res., 103(E9):19963-19972, 1998.

O. P. Makarov, I. Kanik, and J. M. Ajello. Electron impact dissociative excitation of $\mathrm{O}_{2}$ : 1 . Kinetic energy distributions of fast oxygen atoms. J. Geophys. Res., 108, 2003.

M. L. Marconi. A kinetic model of Ganymede's atmosphere. Icarus, 190:155-174, 2007.

N. J. Mason and W. R. Newell. Electron impact dissociation of $\mathrm{O}_{2}$. J. Phys. B. At. Mol. Opt. Phys., 23:4641-4653, 1990.

H. G. Mayr and H. Volland. Magnetic storm effects in the neutral composition. Planet. Space Sci., 20:379-393, 1972.

W. E. McClintock, G. J. Rottman, and T. N. Woods. Solar-Stellar Irradiance Comparison Experiment II (SOLSTICE II): Instrument Concept and Design. In G. Rottman, T. Woods, and V. George, editors, The Solar Radiation and Climate Experiment (SORCE), pages 225258. Springer New York, 2005.

M. A. McGrath, E. Lellouch, D. F. Strobel, P. D. Feldman, and R. E. Johnson. Satellite Atmospheres. In F. Bagenal, T. Dowling, and W McKinnon, editors, Jupiter - the planet, satellites and magnetosphere, chapter 19. Cambridge University Press, 2004.

M. A. McGrath, C. Hansen, and A. Hendrix. Observations of Europa's Tenuous Atmosphere. In R. Pappalardo, W. McKinnon, and K. Khurana, editors, Europa. University of Arizona Press, 2009.

M. A. McGrath, X. Jia, K. Retherford, P. D. Feldman, D. F. Strobel, and J. Saur. Aurora on ganymede. J. Geophys. Res., 118:1-12, 2013.

S. B. Mende, H. Heetderks, H. U. Frey, J. M. Stock, M. Lampton, S. P. Geller, R. Abiad, O. H. W. Siegmund, S. Habraken, E. Renotte, C. Jamar, P. Rochus, J.-C. Gérard, R. Sigler, and H. Lauche. Far ultraviolet imaging from the IMAGE spacecraft. 3. Spectral imaging of Lyman- $\alpha$ and OI 135.6 nm. Space Sci. Rev., 91:287-318, 2000. 
J. S. Murphree, R. A. King, T. Payne, K. Smith, D. Reid, J. Adema, B. Gordon, and R. Wlochowicz. The Freja ultraviolet imager. Space Sci. Rev., 70:421-446, 1994.

R. Prangé, D. Rego, L. Pallier, J. E. P. Connerney, P. Zarka, and J. Queinnec. Detailed study of FUV Jovian auroral features with the post-COSTAR HST faint object camera. J. Geophys. Res., 103(E9):20195-20215, 1998.

F. L. Roesler, H. W. Moos, R. J. Oliversen, R. C. Woodward Jr., K. D. Retherford, F. Scherb, M. A. McGrath, W. H. Smyth, P. D. Feldman, and D. F. Strobel. Far-ultraviolet imaging of Io's atmosphere with HST/STIS. Science, 283:353-357, 1999.

L. Roth, J. Saur, K. D. Retherford, D. F. Strobel, P. D. Feldman, M. A. McGrath, and F. Nimmo. Transient water vapor at europa's south pole. Science, 343:171-174, 2014.

G. J. Rottman, T. N. Woods, and T. P. Sparn. Solar-Stellar Irradiance Comparison Experiment 1: 1. Instrument Design and Operation. J. Geophys. Res., 98:10667-10677, 1993.

J. Saur, P. D. Feldman, L. Roth, F. Nimmo, D. F. Strobel, K. D. Retherford, M. A. McGrath, N. Schilling, J.-C. Gèrard, and D. Grodent. Hubble Space Telescope/ Advanced Camera for Surveys observations of Europa's atmospheric ultraviolet emission at eastern elongation. Astrophys. J., 738, 2011.

A. J. Steffl, F. Bagenal, and A. I. F. Stewart. Cassini UVIS observations of the Io plasma torus. 2. Radial variations. Icarus, 172:91-103, 2004.

M. H. Stevens, J. Gustin, J. M. Ajello, J. S. Evans, R. R. Meier, A. J. Kochenash, A. W. Stephan, A. I. F. Stewart, L. W. Esposito, W. E. McClintock, G. Holsclaw, E. T. Bradley, B. R. Lewis, and A. N. Heays. The production of Titan's ultraviolet nitrogen airglow. J. Geophys. Res., $116,2011$.

D. F. Strobel and D. E. Shemansky. EUV emission from Titan's upper atmosphere: Voyager 1 encounter. J. Geophys. Res., 87:1361-1368, 1982.

D. F. Strobel, R. R. Meiers, M. E. Summers, and D. J. Strickland. Nitrogen airglow sources: Comparison of Triton, Titan and Earth. Geophys. Res. Lett., 18:689-692, 1991.

D. F. Strobel, J. Saur, P. D. Feldman, and M. A. McGrath. Hubble Space Telescope Space Telescope Imaging Spectrograph search for an atmosphere on Callisto: A jovian unipolar inductor. Astrophys. J., 581:L51 - L54, 2002. 
M. R. Torr, D. G. Torr, M. Zukic, R. B. Johnson, J. Ajello, P. Banks, K. Clark, K. Cole, C. Keffer, G. Parks, B. Tsurutani, and J. Spann. A far ultraviolet imager for the international solar-terrestrial physics mission. Space Sci. Rev., 71:329-383, 1995.

J. H. Waite Jr., G. R. Gladstone, W. S. Lewis, R. Goldstein, D. J. McComas, P. Riley, R. J. Walker, P. Robertson, S. Desai, J. T. Clarke, and D. T. Young. An auroral flare at jupiter. Nature, 410:787-789, 2001.

J. R. Walsh. Extraction of Point Source Spectra from STIS . In 1997 HST Calibration Workshop, pages 156-160, 1997.

W. L. Wiese, J. R. Fuhr, and T. M. Deters. Atomic transition properties of carbon, nitrogen and oxygen - a critical data compilation. AIP press, 1996.

D. J. Williams, B. H. Mauk, R. W. McEntire, E. C. Roelof, T. P. Armstrong, B. Wilken, J. G. Roederer, S. M. Krimgris, T. A. Fritz, L. J. Lanzerotti, and N. Murphy. Energetic particle signatures at ganymede: Implications for ganymede's magnetic field. Geophys. Res. Lett., 24: 2163-2166, 1997.

M. Zastrow, J. T. Clarke, A. R. Hendrix, and K. S. Noll. UV spectrum of Enceladus. Icarus, $220: 29-35,2012$.

M. Zukic, D. G. Torr, J. Kim, J. F. Spann, and M. R. Torr. Filters for the International Solar Terrestrial Physics Mission far-ultraviolet imager. Opt. Eng., 32:3069-3074, 1993. 